\title{
Tobacco Use in Bipolar Disorder
}

\author{
Daniel Thomson', Michael Berk ${ }^{2,3,4}$, Seetal Dodd ${ }^{2,4}$, Marta Rapado-Castro ${ }^{5,6,7}$, Shae E. Quirk ${ }^{2}$, Pernille K. \\ Ellegaard $^{8}$, Lesley Berk ${ }^{9}$, Olivia M. Dean ${ }^{2-4}$
}

'Department of Applied Sciences, RMIT, Bundoora, ${ }^{2}$ IMPACT Strategic Research Centre, School of Medicine, Deakin University, Barwon Health, Geelong, ${ }^{3}$ Florey Institute for Neuroscience and Mental Health, University of Melbourne, Parkville, ${ }^{4}$ Department of Psychiatry, University of Melbourne, Parkville, ${ }^{5}$ Orygen Youth Health Research Centre, Centre for Youth Mental Health, University of Melbourne, Victoria, ${ }^{6}$ Melbourne Neuropsychiatry Centre, Department of Psychiatry, The University of Melbourne and Melbourne Health, Victoria, Australia, ${ }^{7}$ Department of Child and Adolescent Psychiatry, Hospital General Universitario Gregorio Marañón, CIBERSAM, IiSGM, School of Medicine, Universidad Complutense, Madrid, Spain, ${ }^{8}$ Research Unit, Mental Health Services, Region of Southern Denmark, Esbjerg, Denmark, ${ }^{9}$ Centre for Mental Health and Wellbeing Research, School of Psychology, Deakin University, Burwood, Australia

\begin{abstract}
Tobacco use in mental health in general and bipolar disorder in particular remains disproportionally common, despite declining smoking rates in the community. Furthermore, interactions between tobacco use and mental health have been shown, indicating the outcomes for those with mental health disorders are impacted by tobacco use. Factors need to be explored and addressed to improve outcomes for those with these disorders and target specific interventions for people with psychiatric illness to cease tobacco smoking. In the context of bipolar disorder, this review explores; the effects of tobacco smoking on symptoms, quality of life, suicidal behaviour, the biological interactions between tobacco use and bipolar disorder, the interactions between tobacco smoking and psychiatric medications, rates and factors surrounding tobacco smoking cessation in bipolar disorder and suggests potential directions for research and clinical translation. The importance of this review is to bring together the current understanding of tobacco use in bipolar disorder to highlight the need for specific intervention.
\end{abstract}

KEY WORDS: Bipolar disorder; Smoking; Smoking cessation; Quality of life; Psychotropic drugs.

\section{INTRODUCTION}

Tobacco smoking is a leading preventable cause of poor health and premature mortality. ${ }^{1)}$ While tobacco smoking rates in the general population are declining, they remain raised in cohorts with psychiatric problems. ${ }^{2)}$ Nicotine, the primary active compound found in tobacco, is highly addictive. ${ }^{3)}$ High rates of tobacco smoking have been described in bipolar disorder and are associated with poorer outcomes. ${ }^{4)}$ Tobacco smoking is approximately 2-3 times more prevalent in bipolar disorder than in the general population, with estimates ranging from $60-70 \%$ in bipolar patients compared to $25-30 \%$ in the general population. ${ }^{2,45)}$ This elevated smoking rate may not only impact on the course of the disorder but may also contribute to higher levels of tobacco-related morbidity and mortality.

\footnotetext{
Received: June 16, 2014 / Revised: July 22, 2014

Accepted: July 23, 2014

Address for correspondence: Olivia M. Dean, PhD

IMPACT Strategic Research Centre, School of Medicine, Deakin

University, P. O. Box 281, Geelong, Victoria 3220, Australia

Tel: +61-3-4215-3300, Fax: +61-3-4215-3491

E-mail: oliviad@barwonhealth.org.au
}

Despite high rates of comorbidity and related morbidity, there is a only modest field of research focusing on smoking among individuals with bipolar disorder and the relationship with important clinical and public health outcomes including: the effects on mood symptoms, quality of life, suicidal behaviour, implications for pharmacological treatment, barriers to cessation, and the biological pathways modulating the expression of pathophysiology. Moreover, results from existing studies spanning these topics appear conflicting, suggesting uncertainly exists in the literature.

Some authors have argued that tobacco smoking results in poorer treatment outcomes in bipolar disorder, ${ }^{7-12}$ ) whereas other researchers have not found such a relationship. ${ }^{13,14)}$ Variance between studies may be due to design factors including the sample size, the sample selection inconsistent measurements of smoking behaviour, and the methodological design of the studies. Given this, the following review aims to explore the issues specific to smoking cessation in bipolar disorder and provide the most clarity regarding treatment options and outcomes.

A comprehensive search using PubMed and Google

(c) This is an Open-Access article distributed under the terms of the Creative Commons Attribution Non-Commercial License (http://creativecommons.org/licenses/by-nc/3.0) which permits unrestricted non-commercial use, distribution, and reproduction in any medium, provided the original work is properly cited. 
Scholar was conducted using the terms "smoking and mania", "smoking and bipolar disorder" and "nicotine dependence and bipolar disorder". The purpose of the current review was to explore the effects of tobacco smoking in bipolar disorder, examining the relationship between smoking and mood symptoms (e.g., depression and mania), quality of life, suicidal behaviour, biological interactions between the pathophysiology of the disorder and the addiction to tobacco, as well as the effects of tobacco smoke on medication and of smoking cessation. Specifically, this paper discusses pertinent studies spanning clinical and public health topics, addressing current gaps in the field and extending previous reviews conducted, with an aim of prompting further research in the nascent field. Such an evidence base may also provide a context for guiding policy makers and informing clinical practice.

\section{MAIN SUBJECTS}

\section{Tobacco Smoking Cessation and Effects on Mood Symptoms}

At a population level, mood symptoms such as mania or depression appear to be associated with tobacco use. The presence of mania and hypomania is associated with 3.9 and 3.5 times greater likelihood of tobacco dependence in the psychiatric population, respectively. ${ }^{15)}$ Additionally, during acute tobacco smoking cessation, those with bipolar disorder may have higher risks of experiencing mania, ${ }^{16,17)}$ and transient depressive recurrence. ${ }^{18)}$ Furthermore, Adan et al. ${ }^{19)}$ have reported low mood scores in highly dependent smokers, intermediate mood scores in low dependent smokers and high mood scores in non-smokers, suggesting the role nicotine dependence plays in mood regulation. Another issue regarding bipolar disorder and tobacco smoking cessation is the potential effect of cessation on mood symptoms. Although there have been case reports of mania, ${ }^{16,17)}$ and depression, ${ }^{18)}$ following abrupt nicotine withdrawal, there are few double blind placebo controlled studies that have looked at the effect of tobacco cessation on mood. A key recent meta-analysis has shown that on aggregate, mental health symptoms, particularly depression, improve in those who quit smoking. ${ }^{20)}$

Those with bipolar disorder might thus be proposed to have an increased susceptibility to tobacco smoking despite the observation that many patients start to smoke before the onset of their illness. ${ }^{21-23)}$ Research shows that most smokers take up the habit as teenagers, ${ }^{24)}$ with recent statistics in Australia revealing that in 2010, the age of ini- tiation was $16 .{ }^{25)}$ Regarding the age of onset in bipolar disorder, for bipolar type I the average age of onset is 23 years for males and 26 years for females, and for bipolar type II, the age of onset is 29.7 years for males and 30.1 years for females. ${ }^{26)}$ A line of research has postulated that enduring alterations in neurophysiology as a result of tobacco abuse may unmask an underlying vulnerability to affective disorders, including bipolar disorder. ${ }^{19,27,28)}$ However, the mechanisms underlying the relationship between tobacco smoking and bipolar disorder are clearly bidirectional, complex and multifactorial. ${ }^{13,29)}$ Therefore, while it is probably correct that in most instances tobacco smoking behaviour pre-dates and is a risk factor for bipolar disorder, caution is recommended when interpreting these findings and implying causality.

Specific associations between tobacco smoking status and symptom characteristics of the disorder have been described..$^{7,8,11,12,30,31)}$ In an early study looking at the role of tobacco smoking and psychotic symptom status in bipolar disorder, Corvin et al. ${ }^{12)}$ examined the effect of smoking severity on a sample of 92 patients with bipolar disorder. Tobacco smoking was less prevalent in less symptomatic patients whereas pervasiveness of smoking was correlated with severity of symptoms (particularly psychotic symptoms). This was a cross-sectional study with a small sample size using self-report measures which should be noted when interpreting the results, as no objective measure such as blood test were used to detect nicotine use. A recent prospective and naturalistic study over 24 months examined the effect of tobacco smoking on mental health outcomes in 239 patients with schizoaffective and bipolar disorder, ${ }^{11)}$ and found that in both schizoaffective and bipolar disorder, daily smokers had poorer prognosis in terms of depression and overall bipolar symptomatology (assessed using the Clinical Global Impressions - Depression and Clinical Global Impressions - Overall Bipolar scales) and longer stays in hospital compared with non-smokers. An important limitation, similar to Corvin and colleagues, ${ }^{12)}$ is that the study did not objectively measured nicotine dependence and used self-report measures. This is again an important issue as varying levels of nicotine dependence may affect overall scores and mood symptomatology more than simply assessing daily smoking rates reported by the patients. Daily smoking rates do not necessarily address smokers' levels of nicotine addiction amongst patients with bipolar disorder.

Other studies have reported a link between tobacco smoking and worse outcomes in both mania and depression in bipolar disorder. ${ }^{7,8,10,30,31)}$ In particular, a large 
study of 399 patients with bipolar disorder reported that a lifetime history of tobacco smoking was significantly related to earlier onset of the first depressive or manic episode, greater symptom severity, poorer functioning, a lifetime history of suicide attempt comorbid anxiety and substance abuse. ${ }^{10)}$ In addition, results from the Systematic Treatment Enhancement Program for Bipolar Disorder (STEP-BD) have linked tobacco smoking to clinical variables including more lifetime manic and depressive symptoms, rapid cycling illness, comorbid mental disorders, substance abuse and active illness. ${ }^{8)}$ However cross-sectional studies have showed inconsistent findings. ${ }^{13,14)}$ In the most recent study, illness severity, number of episodes, history of psychotic symptoms, history of suicide attempts, age at onset of the illness, abuse of psychoactive drugs, current clinical status and other demographic variables and their relationship with tobacco smoking were examined in a sample of 102 participants with bipolar disorder. There was a higher tobacco smoking prevalence in this population and a higher presence of alcohol and other drugs use and earlier age of illness onset in those patients with bipolar disorder who smoked more. No other association with regard to illness severity was found in this study. However, the authors caution against generalisation due to the small sample size and the cross sectional study design. Furthermore, nicotine levels were not measured in this study. Similarly, Cassidy et al. ${ }^{13)}$ reported no association between smoking and psychosis in patients with bipolar disorder, but no measures of mood were included in the analysis.

Inconsistent findings on the impact of tobacco smoking on prognosis in bipolar disorder have also been recently reported in a comprehensive review on the effects of smoking on the health outcomes in bipolar disorder. ${ }^{32)}$ In the reviewed studies, tobacco smoking was correlated with rapid cycling, ${ }^{4,8,33)}$ more affective episodes, ${ }^{32,33)}$ elevated risk of psychotic episodes, ${ }^{12)}$ and more time spent in hospital, ${ }^{20,30)}$ in some studies but not in others. Individual differences in nicotine dependence may account for the variability of the findings between studies. Methodological complexities including limited number of participants, bipolar patients who are nicotine dependent versus smokers who are non-nicotine dependent, admixtures of prospective and cross-sectional studies, diverse measures and lack of objective biological markers might account for the difference observed.

\section{Quality of Life and Tobacco Smoking in Bipolar Disorder}

Tobacco use enhances mortality and decreases quality of life through a number of smoking-related health diseases such as cardiovascular, cancer or respiratory conditions. $^{34)}$ Tobacco smoking has also been related to more severe course, relapse and higher number of hospitalizations, ${ }^{20)}$ and is also associated with an elevated risk of concomitant substance use disorders. ${ }^{24)}$ Few studies have examined the quality of life in smokers versus non-smoker with bipolar disorder. Consistent findings across studies have shown that quality of life is higher in non-smokers and occasional smokers compared with nicotine dependent bipolar patients. ${ }^{11,21,22)}$ Smoking has also been associated with low socioeconomic status in patients with bipolar disorder, ${ }^{8)}$ and with the risk of anxiety disorders in the general population, ${ }^{23)}$ which may be moderating or mediating pathways.

In a study examining the quality of life in bipolar disorder in comparison with that of the general population, 48 euthymic and 60 non-euthymic bipolar patients were compared with 1210 controls using the physical and mental component scores of the Medical Outcomes Survey 36-item Short Form Health-Survey (SF-36). ${ }^{21)}$ Clinical and course of illness variables and their association with low physical and mental quality of life were explored including manic and depressive symptoms and consumption of other addictive substances. Lower mental quality of life was associated with longer duration of illness and early onset in bipolar patients. A higher presence of depressive symptoms, nicotine dependence and lack of social support was also reported in the bipolar group. Further studies have replicated this association. Quality of life in terms of physical health, psychological wellbeing and environmental and social relationships was lower in bipolar patients with nicotine dependence than in those without nicotine dependence. ${ }^{22)}$ Worse health related quality of life scores were also found for daily smokers. ${ }^{11)}$

It is plausible that tobacco smoking in bipolar disorder might be indirectly influencing this construct through modifiable lifestyle factors that have also shown to be related to bipolar disorder. Poorer diet and lower levels of physical activity have been associated with smokers, in addition to these factors already being present in bipolar disorder. $^{24-27)}$ Furthermore, tobacco smoking may play a detrimental role in sleep quality for those with bipolar disorder. Sleep disturbance has comprehensively and independently been associated with poor health-related quality of life. ${ }^{35)}$ In a study including 252 participants with mood disorder, the severity of the disorder was positively associated with the mean number of cigarettes smoked per day and with greater levels of fatigue. Additionally, mild 
to severe symptoms of insomnia were related to the level of nicotine dependence. ${ }^{28)}$ Differences between smokers and non-smokers in circadian rhythmicity and mood variation have been reported in the general population. ${ }^{19)}$ Altered sleep patterns play a detrimental role in bipolar disorder, ${ }^{29)}$ as disrupted night time sleep and day time mood have been proposed to be mutually reinforcing. ${ }^{36)}$ This bidirectional model, known as the sleep-mood cycle, is of particular importance to bipolar disorder and insomnia. ${ }^{36)}$ Disruptions in circadian rhythms have been identified as a major trigger for the onset and relapse of bipolar disorder. ${ }^{37-40)}$ These modifiable lifestyle factors not only play a role in quality of life for bipolar disorder, but these relationships imply that they could play a critical part in the management of the illness.

\section{The Relationship between Tobacco Smoking and Suicidal Behaviour in Bipolar Disorder}

To date, many studies have identified associations between psychiatric disorders, comorbidities and suicide attempts. This has been demonstrated across several studies utilizing adequate study designs with large sample sizes. ${ }^{41)}$ In particular, tobacco smoking has also been independently linked to increased risk of suicidality, an association that was sustained after adjustment for psychiatric disorders. ${ }^{42)}$

Individuals with bipolar disorder, have higher rates of suicide than the general population. ${ }^{43)}$ Moreover, the contribution of disability and mortality related to physical causes of death, suicide and injuries incurred via self-harm in this group are significant. ${ }^{10)}$ Due to the deleterious outcomes for individuals and significant burden that both tobacco smoking and suicidal behaviour incurs, it is necessary to understand the role of this potentially modifiable lifestyle factor and its link to suicidal behaviour in this group to reduce mortality and disability.

Several studies have demonstrated associations between tobacco smoking in bipolar disorder and suicidal behaviour. ${ }^{8,10,33,44,45)}$ In one study, predictors of suicidal acts were investigated in a 2-year follow-up study of patients $(n=308)$ presenting with major depressive disorder or bipolar disorder at baseline. ${ }^{44)}$ Cox proportional hazards regression analyses revealed that tobacco smoking was among the strongest predictors for future suicidal acts, alongside history of suicidal acts and self-reported severity of depression. Furthermore, among other significant clinical predictors for suicidal acts were presence of a comorbid Cluster B personality disorder, and aggressive and impulsive personality traits. These person- ality features and traits have been shown to be risk factors for self-mutilation, help-seeking suicide threats and/or attempts and completed suicide, ${ }^{46-48)}$ and frequently co-occur with bipolar disorder. ${ }^{49)}$ It is possible that comorbidity among impulsive/aggressive features and smoking increases the risk for suicidal behaviours in bipolar disorder.

A retrospective clinical study (1999-2004) also demonstrated significant associations between lifetime tobacco smoking and suicide attempts in patients with bipolar disorder, after adjusting for other psychiatric comorbidities and measures of disability and functional impairment. ${ }^{10)}$ Finally, subsequent prospective analyses have also reported that tobacco smoking was associated with increased risk of suicidality in individuals with bipolar disorder $(\mathrm{n}=106)$, throughout the course of a nine-month follow-up. ${ }^{35)}$ These baseline associations were not sustained after adjustment for impulsivity in the models. Moreover, small sample sizes precluded further analyses examining associations between impulsivity, smoking and suicide attempts.

The increased risk for suicide attempts among those with bipolar disorder, and who smoke, appears to true even for youths. The Course and Outcome of Bipolar Youth Study examined 212 youths (7-17 years) with bipolar disorder and correlates. Differences between three groups of tobacco smoking status (daily, never smoked, ever smoked) were observed, with daily smokers having a higher lifetime prevalence of suicide attempts. Logistic regression found those with a history of tobacco smoking had approximately a three-fold increased risk of lifetime suicide attempt compared to those without. However, it was beyond the scope of the study to examine the prevalence and correlates of nicotine dependence among the youths with bipolar disorder. Further, longitudinal analyses of subsequent follow-up visits may elucidate temporal relationships smoking and suicidality in the younger with bipolar disorder.

Contrary these findings, others have reported no significant associations for tobacco smoking and suicidality in bipolar disorder in longitudinal analyses. ${ }^{50-52)}$ In one study, $40.5 \%$ of those who had attempted and/or completed suicide over the observational period ( 2 years) were smokers. ${ }^{48)}$ Whilst there was an initial trend for associations between baseline tobacco smoking status and increased risk for suicide attempts, the association was not sustained in logistic regression models, ${ }^{52)}$ as was the case in another study with a similar follow-up period. ${ }^{51)} \mathrm{An}$ additional prospective study also reported that tobacco smoking did not predict suicide attempts over the ob- 
servational period (18 months). ${ }^{50)}$ A review examining the clinical and health outcomes of a number of psychiatric disorders, also suggested that associations between tobacco smoking and suicidal behaviour in patients with bipolar disorder was inconclusive. ${ }^{32)}$

These studies highlight that tobacco smoking may be independently associated with suicide attempts in individuals with bipolar disorder. However, there remains areas of uncertainty about the relationship between tobacco smoking and bipolar disorder in regards to suicidal behaviour. It may be that personality variables (linked to impulsivity) may be important effect modifiers in the relationship between tobacco smoking and suicidality, particularly during acute phases of the disorder. Examining the potential role of personality as a mediating and/or moderating variables in future studies examining tobacco smoking and comorbidities is warranted.

\section{Biological Interactions between the Pathophysiology of Bipolar Disorder and Tobacco Addiction}

To date, operative mechanisms linking tobacco smoking and bipolar disorder remain unclear. In unipolar depression, the role of neurotransmitters has been hypothesized. Associations may be related to lower levels of brain serotonergic function in smokers. ${ }^{53)}$ Lower levels of serotonin in both humans and primates have been linked with higher levels of impulsive, novelty seeking behaviour. ${ }^{54)}$ However, given discrepancies between the underlying biology in unipolar and bipolar depression (see next section), caution needs to be taken when considering these links. Additionally, dysregulation of the dopamine and glutamatergic systems and their inputs to different regions of the brain (particularly prefrontal cortex and basal ganglia) have been linked to the maintenance and relapse of addictive behaviour. ${ }^{55-57)}$ Nicotine is a nicotinic cholinergic receptor agonist that causes the release of both dopamine and norepinephrine. These two neurotransmitters are implicated in the pathophysiology of bipolar disorder, and are also thought to play a key role in addiction. This may influence susceptibility of people with bipolar disorder to the rewarding effects of nicotine. Furthermore, smokers have reduced monoamine oxidase which is a potential pharmacological effect of tobacco smoke as it prolongs the synaptic presence of neurotransmitters involved in mood regulation (i.e., serotonin and dopamine) ${ }^{58)}$ Cholinergic- monoamine pathway interactions may link nicotine dependence and bipolar disorder given the putative role of dopamine, serotonin norepinephrine and glutamine, ${ }^{4,59)}$ in the pathogenesis of both bipolar disorder and nicotine addiction and the role of monoamine neurotransmitters in mood disorders.

In this regard, cyclical dopamine dysregulation has been proposed as a central mechanism underlying bipolarity. ${ }^{60)}$ The activation of the mesolimbic dopaminergic pathways through the diffuse cholinergic innervations in the brain is thought to play a salient role in the generation of reward and reinforcement of tobacco smoking. ${ }^{61)}$ Further to this, dynamic desensitization of nicotinic cholinergic, dopaminergic and other downstream receptors may account not only for nicotine tolerance and withdrawal symptoms but also for smoking conditioning through the rewarding effects of nicotine in the brain. An increase in dopaminergic transmission would predominate during the manic phase there is whereas a secondary down regulation of receptors would eventually dampen the cycle learning to a phase of decreased dopaminergic transmission, characteristic of the depressive phase. ${ }^{60)}$ This theory of the role of the dopaminergic system provides support for the relationship between tobacco smoking and mood disorders. There might be a number of other underlying neurobiological mechanisms to account for this relationship given the vast array of other neurochemicals triggered by nicotine. Enduring changes in excitatory glutamatergic synapses on the nucleus accumbens are induced by nicotine use ${ }^{55,62)}$ and have been shown to be involved in drug relapse. ${ }^{63)}$ Recently, disturbances in glutamatergic function have also been implicated in the pathophysiology of mood disorders (major depressive disorder and bipolar disorder). ${ }^{46-49)}$ The role of inflammation and oxidative and nitrosative stress as a plausible mediator for the development of depressive and bipolar disorders and its relationship to tobacco use have also been examined in recent years. The literature also provides some support for a potential interaction between tobacco smoking and the neuroprogressive course of the disorders as these pathways are affected by exposure to tobacco smoke components such as nicotine and free radicals. ${ }^{64-66)}$

\section{Effects of Tobacco Smoking on Pharmacological Treatment in Bipolar Disorder}

The effect of tobacco smoking on the impact of the metabolism of many psychotropic medications used in the treatment of bipolar disorder is well documented. ${ }^{4,11,67)}$ Polycyclic aromatic hydrocarbons in tobacco smoke, increase the metabolism of some psychotropic medications, including olanzapine, clozapine, haloperidol and fluvoxamine.) ${ }^{4)}$ This is largely mediated via the induction of the 
hepatic cytochrome P450 enzyme 1A2 by the polycyclic aromatic hydrocarbons found in tobacco smoke, leading to lower serum levels of drugs including olanzapine as well as other antipsychotics, antidepressants and benzodiazepines. This may be a pathway whereby tobacco smoking reduces the therapeutic benefits of taking these medications. This results in smokers with bipolar requiring an increased dose, approximately $50 \%$ higher, to achieve an adequate therapeutic response. ${ }^{67)}$

Additionally, some case reports suggests that a substantial increase in tobacco smoking can lead to an increase in psychiatric symptoms among individuals with bipolar disorder who are taking olanzapine, at least in part due to the reduction in medication levels explored the dose dependent effect of tobacco smoking on serum concentrations of clozapine and olanzapine ${ }^{67)}$ The mean serum concentration to dose ratio was twice as high in non-smokers compared to smokers for both olanzapine and clozapine, leading to authors to consider differential starting doses in smokers compared to non-smokers.

It has been further reported that nicotine's activation of the sympathetic nervous system may reduce the sedating effects of benzodiazepines and the capacity of beta blockers to lower heart rate and blood pressure. ${ }^{4)}$ The evidence that tobacco smoking not only interferes with a range of different medications, points to the widespread effect that nicotine and tobacco smoke plays on multiple neurotransmitter and brain systems. ${ }^{11,67-69)}$ Dani and De Biasi ${ }^{68)}$ suggest fast nicotinic transmission is used throughout the peripheral nervous system and at the neuromuscular junctions, leading to body-wide impacts. This view is supported by Laviolette and van der Kooy, ${ }^{69)}$ who state that nicotine produces diverse neurophysiological, motivational and behavioural effects throughout several brain regions via multiple neurochemical pathways.

The far-reaching ability of nicotine to produce changes in multiple neurotransmitter systems is possibly through the widespread cholinergic innervations that nicotine plays on the brain. As nicotine is a nicotinic cholinergic receptor agonist it enhances the release of a cascade of neurotransmitters including dopamine, serotonin, norepinephrine and glutamine. Agents that drive dopamine such as cocaine and amphetamine are among the best models of mania, while dopamine D2 antagonists are robustly anti-manic and include several antipsychotic agents. ${ }^{61)}$ Nicotine may thus indirectly drive dopamine itself as well as counteract the effects of pharmacological interventions, firstly via its psychostimulant properties, and secondly via pharmacokinetic interactions as highlighted above

Hutchison et $_{\mathrm{al}}{ }^{70)}$ looked at the atypical antipsychotic, olanzapine, in attenuating cue elicited craving for tobacco. The hypothesis was that if mesolimbic dopaminergic activity is associated with cue-elicited cravings for tobacco, a dopaminergic antagonist should attenuate cue-elicited cravings for tobacco. Olanzapine attenuated cue elicited craving for tobacco, but did not moderate the subjective effects of tobacco smoking in a randomly assigned five day placebo controlled trial. However, dopamine antagonists decrease the pleasure, reinforcement and reward of tobacco smoking, which might lead to increase smoking behaviour. ${ }^{71)}$

\section{Tobacco Smoking Cessation and Bipolar Disorder}

The rate of successful cessation attempts for people with mental illness is low. Data from the United States 2002 National Survey on Drug Use and Health demonstrated that the quit rate was $49 \%$ for former smokers with no serious psychological distress, determined using the K6 scale, whereas the quit rate was $29 \%$ for former smokers with serious psychological distress. ${ }^{72)}$ The reason for these reduced quit rates has been examined by tobacco control researchers, with one view describing the concept of 'hardening' and 'hard-core smokers', where reduced quit rates are attributed to greater difficulty in quitting or less willingness to do so. ${ }^{73)}$ In the general population, evidence of hardening remains elusive as tobacco smoking rates continue to decline; however, amongst people with bipolar and other major psychiatric disorders smoking rates have not declined at the same rate and hardening may be a factor. However, it is very unlikely to fully explain the disparity in quit rates between the mentally ill and general populations. Smokers with mental illness may be as motivated to quit smoking tobacco as smokers without a current mental illness. This has been demonstrated in studies of an inpatient population ${ }^{58)}$ in Australia. There is some data indicating less readiness to quit amongst bipolar patients in India ${ }^{74)}$; however, this may be attributed to a lesser awareness of the health risks of tobacco smoking in that specific population. This suggests that willingness to quit may not be a factor in the discrepancies between quit rates between mentally ill and not mentally ill smokers. Greater difficulty in quitting smoking may still be a 'hardening' factor in this population as most readily available tobacco smoking cessation strategies have been developed for the general population and may not be as effective for mentally ill smokers.

Other non-hardening factors may also be applicable to 
this population, including less awareness of the health risks associated with tobacco smoking, a factor likely to vary considerably between and even within different countries. There are reports that some people with mental illness may feel alienated from approaching mainstream tobacco smoking cessation services. ${ }^{8)}$ However, the reasons for the disparity in smoking cessation rates between mentally ill and not mentally ill people have not been sufficiently researched.

In a review of tobacco smoking cessation in bipolar disorder, George et al ${ }^{33)}$ found just 11 clinical studies on bipolar disorder and tobacco addiction showing that people with bipolar disorder have much lower quit rates than smokers without a comorbid condition. Several case reports as reviewed by Dome et $a l .{ }^{32)}$ suggested that varenicline treatment, for tobacco cessation, may provoke mania in patients with or without a history mood disorders. However this has not been borne out by large scale well designed randomized trials. ${ }^{60)}$

There have only been four published controlled smoking cessation treatment studies in bipolar smokers. The first two are relatively small studies, with two recent more definitive studies with varenicline. The initial small pilot study $(n=5)$ but showed buproprion was well-tolerated and potentially useful during 10 weeks of treatment. ${ }^{75)}$ Following on from this, Wu et al ${ }^{76)}$ conducted another tiny $(n=5)$ study of varenicline for tobacco cessation in those with bipolar disorder. Over the 10-week treatment phase, reduction in tobacco smoking in the varenicline group was found and it was well tolerated. In the first large randomized controlled trial of varenicline for tobacco cessation maintenance in individuals diagnosed with bipolar disorder and schizophrenia, 87 participants were included in the maintenance phase, of which only 13 were diagnosed with bipolar disorder (the remaining were diagnosed with schizophrenia). The study included an open-label phase for 12 weeks following which participants who were abstinent for at least 14 days were then randomized to receive either continued varenicline treatment, or placebo up to 52 weeks of treatment. ${ }^{60)}$ Varenicline was efficacious as a maintenance treatment in the study, and this effect remained when those with bipolar disorder were analysed separately. The largest and most rigorous study was that by Evins et al., ${ }^{60)}$ who enrolled 247 smokers with schizophrenia or bipolar disorder from 10 community mental-health centres. Of these, 203 received 12-week treatment with both open-label varenicline and cognitive behavioral therapy, after which 87 met abstinence criteria and entered the relapse prevention in- tervention. After a year, the point-prevalence abstinence rates were $60 \%$ with varenicline treatment and $19 \%$ with placebo, giving an odds ratio of 6.2. Importantly, there were no impacts on psychiatric symptoms.

This paper has highlighted that tobacco smoking is deleterious to mental health, but a critical question is whether mental health benefits from tobacco smoking cessation. A critical review and meta-analysis has recently been published, with striking findings. Firstly, quitting has been linked to reduced anxiety and stress, depression, and improved positive mood as well as quality of life compared with ongoing tobacco smoking. Secondly, effect sizes are equivalent for people with psychiatric disorders compared to those without. Lastly, effect sizes appear similar or greater than those of antidepressant treatment for the abovementioned disorders. ${ }^{77)}$ This finding highlights the necessity to work towards cessation.

It is not clear how best to assist smokers with mental illness to quit tobacco smoking. There are additional factors that may further contribute to the success or failure of smoking cessation in those particularly, diagnosed with bipolar disorder. Given the high rates of comorbid anxiety disorders and substance abuse the complexities of treatment for smoking cessation are increased. George et al. ${ }^{33)}$ have suggested that the alternating mood fluctuations (ranging from irritable and elevated mood to severe depression) may contribute to differences in smoking cessation rates found in bipolar disorder. Similarly, Ostacher et $a l{ }^{10)}$ reported a relationship between comorbid anxiety disorders and substance abuse in lifetime smokers with bipolar disorder, suggesting further difficulty in smoking cessation. Current smoking cessation interventions for individuals with bipolar disorder include several first line pharmacotherapies that include nicotine replacement therapy, bupropion, sustained release and varenicline. ${ }^{3)}$ It has also been reported that olanzapine can assist in cue prompted cravings for tobacco. ${ }^{70)}$ The problem with most of these therapies is that it is unclear how safe and efficient these aids are for people with bipolar disorder. ${ }^{3)}$ Unlike pharmacotherapy, behavioural interventions have been tested but unfortunately, current evidence is insufficient to recommend these approaches as alternatives to standard counselling. ${ }^{3)}$ However, behavioural interventions improve the chances of quitting, especially when used in conjunction with pharmacotherapy. ${ }^{78)}$ Examples of behavioural interventions include cognitive behaviour therapy, mood management interventions and motivational interviewing.

Most large studies use mixed populations that are gen- 
erally heavily weighted towards high prevalence disorders. El-Guebaly et al. ${ }^{79)}$ reviewed 24 studies of tobacco smoking cessation in people with mental disorder, where the majority of interventions combined pharmacotherapies and psychoeducation, and found that quit rates for people with mental illnesses were similar to those expected for the general population. On the basis of these findings, Australian tobacco smoking cessation programs give recommendations for smoking cessation strategies for people with mental illnesses that are largely identical to recommendations given or people without mental illnesses; apart from warning about altered pharmacokinetics of some psychotropic medications, the possibility of withdrawal symptoms being mistaken for symptoms of mental illness and a risk of exacerbation of depressive symptoms. A government funded tobacco smoking cessation program in Brazil identifies people with mental illnesses and substance abuse and tailored their smoking cessation intervention to their needs. ${ }^{80)}$ Kerr and colleagues ${ }^{81)}$ describe barriers to mainstream tobacco smoking cessation programs for people with mental illnesses that include; perceived benefits of smoking to their symptoms of mental illness, fear of illness worsening, social circles where smokers predominate, low self-confidence, health professionals who are not encouraging their cessation efforts and are not trained in tobacco smoking cessation and reluctance to access mainstream smoking cessation services. Furthermore, health professionals are not always aware of the importance of addressing smoking cessation in this vulnerable group. ${ }^{82)}$ While there is insufficient evidence to recommend any specific tobacco smoking cessation technique for people with bipolar disorder, there is sufficient evidence to conclude that tobacco smoking cessation interventions should be tailored to the individual needs and preferences of the person being treated. For some individuals with bipolar disorder mainstream tobacco smoking cessation techniques may be adequate. For other individuals it may be more effective to provide tobacco smoking cessation support delivered through their usual mental health care provider and providing support techniques additional to those provided by mainstream services. Health professionals should encourage smokers with mental illness to cease tobacco smoking.

\section{FUTURE DIRECTIONS FOR RESEARCH: TOBACCO SMOKING IN BIPOLAR DISORDER}

In this review, an attempt has been made to understand the relationship between smoking and bipolar disorder. As
Pasco et al $^{83)}$ note, it is becoming increasingly clear that tobacco smoking is not only harmful to our physical health, but also for our mental health, which may in fact contribute to its onset or aggravate current symptoms.

It is possible that certain individuals are more vulnerable to nicotine's psychostimulant properties than others and researchers have implied that there is the possibility that separate causal mechanisms may operate in a bi-directional manner. ${ }^{84)}$ Many of the studies evaluated did not assess for nicotine dependence, which may play a bigger role on nicotine's effect on mood symptomatology that rates of daily smoking. It is also clear that more studies need to be conducted to accurately assess the consequences that dependent tobacco smoking places on bipolar disorder. It is suggested that consistent measures of mood symptoms as they apply to bipolar disorder are assessed, as well as consistent measures to assess nicotine dependence amongst individuals with bipolar disorder.

In conclusion, people with bipolar disorder have much higher tobacco smoking rates compared to the general population. Furthermore, although the relationship between tobacco smoking and bipolar disorder may be bidirectional and that cause and effect may be difficult to determine, the majority of studies reviewed suggest that tobacco smoking appears to play a mediating role in the severity of bipolar disorder. Clear gaps exist in the literature, and there is a need for researchers to look at formal smoking cessation programs and the relationship between tobacco smoking, insomnia, lifestyle factors and quality of life in bipolar disorder. Caggiula et al. ${ }^{85)}$ suggest, the abuse liability associated with tobacco smoking equals or exceeds that of other addictive drugs. It is also suggested that tobacco smoking is screened by mental health professionals and targeted during routine treatment, with the implementation of more formal cessation programs. ${ }^{86)}$

\section{Acknowledgments}

Dr. Marta Rapado-Castro was supported by Sara Borrell Health Research Fellowship from the Institute of Health Carlos III, Spanish Ministry of Economy and Competitiveness, an Alicia Koplowitz Short-Term Fellowship and a IiSGM Fellowship Award for Short-term Placements from the Health Research Institute from the Hospital Gregorio Marañón (IiSGM) (Madrid, Spain).

\section{REFERENCES}

1. Thun MJ, Apicella LF, Henley SJ. Smoking vs other risk factors as the cause of smoking-attributable deaths: confounding in the courtroom. JAMA 2000;284:706-712. 
2. Ng M, Freeman MK, Fleming TD, Robinson M, DwyerLindgren $\mathrm{L}$, Thomson $\mathrm{B}$, et al. Smoking prevalence and cigarette consumption in 187 countries, 1980-2012. JAMA 2014:311:183-192.

3. Stolerman IP, Jarvis MJ. The scientific case that nicotine is addictive. Psychopharmacology (Berl) 1995;117:2-10.

4. Heffner JL, Strawn JR, DelBello MP, Strakowski SM, Anthenelli RM. The co-occurrence of cigarette smoking and bipolar disorder: phenomenology and treatment considerations. Bipolar Disord 2011;13:439-453.

5. Lasser K, Boyd JW, Woolhandler S, Himmelstein DU, McCormick D, Bor DH. Smoking and mental illness: A population-based prevalence study. JAMA 2000;284:26062610.

6. Chang CK, Hayes RD, Broadbent M, Fernandes AC, Lee $\mathrm{W}$, Hotopf $\mathrm{M}$, et al. All-cause mortality among people with serious mental illness (SMI), substance use disorders, and depressive disorders in southeast London: a cohort study. BMC Psychiatry 2010;10:77.

7. Berk M, Ng F, Wang WV, Tohen M, Lubman DI, Vieta E, et al. Going up in smoke: tobacco smoking is associated with worse treatment outcomes in mania. J Affect Disord 2008;110:126-134.

8. Waxmonsky JA, Thomas MR, Miklowitz DJ, Allen MH, Wisniewski SR, Zhang $\mathrm{H}$, et al. Prevalence and correlates of tobacco use in bipolar disorder: data from the first 2000 participants in the Systematic Treatment Enhancement Program. Gen Hosp Psychiatry 2005;27:321-328.

9. Vanable PA, Carey MP, Carey KB, Maisto SA. Smoking among psychiatric outpatients: relationship to substance use, diagnosis, and illness severity. Psychol Addict Behav 2003; 17:259-265.

10. Ostacher MJ, Nierenberg AA, Perlis RH, Eidelman P, Borrelli DJ, Tran TB, et al. The relationship between smoking and suicidal behavior, comorbidity, and course of illness in bipolar disorder. J Clin Psychiatry 2006;67:19071911.

11. Dodd S, Brnabic AJ, Berk L, Fitzgerald PB, de Castella AR, Filia $\mathrm{S}$, et al. A prospective study of the impact of smoking on outcomes in bipolar and schizoaffective disorder. Compr Psychiatry 2010;51:504-509.

12. Corvin A, O'Mahony E, O'Regan M, Comerford C, $\mathrm{O}^{\prime}$ Connell $\mathrm{R}$, Craddock $\mathrm{N}$, et al. Cigarette smoking and psychotic symptoms in bipolar affective disorder. $\mathrm{Br} J$ Psychiatry 2001;179:35-38.

13. Cassidy F, McEvoy JP, Yang YK, Wilson WH. Smoking and psychosis in patients with bipolar I disorder. Compr Psychiatry 2002;43:63-64.

14. Kreinin A, Novitski D, Rabinowitz D, Weizman A, Grinshpoon A. Association between tobacco smoking and bipolar affective disorder: clinical, epidemiological, cross-sectional, retrospective study in outpatients. Compr Psychiatry 2012; 53:269-274.

15. Grant BF, Hasin DS, Chou SP, Stinson FS, Dawson DA. Nicotine dependence and psychiatric disorders in the United States: results from the national epidemiologic survey on alcohol and related conditions. Arch Gen Psychiatry 2004;61:1107-1115.

16. Benazzi F. Severe mania following abrupt nicotine withdrawal. Am J Psychiatry 1989;146:1641.

17. Labbate LA. Nicotine cessation, mania, and depression. Am J Psychiatry 1992;149:708.

18. Glassman AH. Cigarette smoking: implications for psychiatric illness. Am J Psychiatry 1993;150:546-553.

19. Adan A, Prat G, Sánchez-Turet M. Effects of nicotine dependence on diurnal variations of subjective activation and mood. Addiction 2004;99:1599-1607.

20. Cassidy F, Ahearn EP, Carroll BJ. Substance abuse in bipolar disorder. Bipolar Disord 2001;3:181-188.

21. Gutiérrez-Rojas L, Gurpegui M, Ayuso-Mateos JL, GutiérrezAriza JA, Ruiz-Veguilla M, Jurado D. Quality of life in bipolar disorder patients: a comparison with a general population sample. Bipolar Disord 2008;10:625-634.

22. Singh J, Mattoo SK, Sharan P, Basu D. Quality of life and its correlates in patients with dual diagnosis of bipolar affective disorder and substance dependence. Bipolar Disord 2005;7:187-191.

23. Moylan S, Jacka FN, Pasco JA, Berk M. How cigarette smoking may increase the risk of anxiety symptoms and anxiety disorders: a critical review of biological pathways. Brain Behav 2013;3:302-326.

24. Alsuwaidan MT, Kucyi A, Law CW, McIntyre RS. Exercise and bipolar disorder: a review of neurobiological mediators. Neuromolecular Med 2009;11:328-336.

25. Sylvia LG, Ametrano RM, Nierenberg AA. Exercise treatment for bipolar disorder: potential mechanisms of action mediated through increased neurogenesis and decreased allostatic load. Psychother Psychosom 2010;79:87-96.

26. Jacka FN, Pasco JA, Mykletun A, Williams LJ, Hodge AM, O'Reilly SL, et al. Association of Western and traditional diets with depression and anxiety in women. Am J Psychiatry 2010;167:305-311.

27. Jacka FN, Rothon C, Taylor S, Berk M, Stansfeld SA. Diet quality and mental health problems in adolescents from East London: a prospective study. Soc Psychiatry Psychiatr Epidemiol 2013;48:1297-1306.

28. Patten CA, Gillin JC, Golshan S, Wolter TD, Rapaport M, Kelsoe J. Relationship of mood disturbance to cigarette smoking status among 252 patients with a current mood disorder. J Clin Psychiatry 2001;62:319-324.

29. Plante DT, Winkelman JW. Sleep disturbance in bipolar disorder: therapeutic implications. Am J Psychiatry 2008; 165:830-843.

30. Yee AHA, Ng CG, Rashid RA, Habil MH. The prevalence and correlates of nicotine use disorder among bipolar patients in a hospital setting, Malaysia. J Dual Diagn 2012; 8:28-34.

31. Chandra PS, Carey MP, Carey KB, Jairam KR, Girish NS, Rudresh HP. Prevalence and correlates of tobacco use and nicotine dependence among psychiatric patients in India. Addict Behav 2005;30:1290-1299.

32. Dome P, Gonda X, Rihmer Z. Effects of smoking on health outcomes in bipolar disorder with a special focus on suicidal behaviour. Neuropsychiatry 2012;2:429-441.

33. George TP, Wu BS, Weinberger AH. A review of smoking cessation in bipolar disorder: Implications for future research. J Dual Diagn 2012;8:126-130.

34. US Department of Health and Human Services. The health consequences of smoking-50 years of progress: A report of the surgeon general, 2014. Rockville, MD:Office of the Surgeon General;2014.

35. Ostacher MJ, Lebeau RT, Perlis RH, Nierenberg AA, Lund HG, Moshier SJ, et al. Cigarette smoking is associated with suicidality in bipolar disorder. Bipolar Disord 2009;11:766771.

36. Talbot LS, Stone S, Gruber J, Hairston IS, Eidelman P, Harvey AG. A test of the bidirectional association between sleep and mood in bipolar disorder and insomnia. J Abnorm Psychol 2012;121:39-50.

37. Chung JK, Lee KY, Kim SH, Kim EJ, Jeong SH, Jung HY, 
et al. Circadian rhythm characteristics in mood disorders: Comparison among bipolar I disorder, bipolar II disorder and recurrent major depressive disorder. Clin Psychopharmacol Neurosci 2012;10:110-116.

38. Jagannath A, Peirson SN, Foster RG. Sleep and circadian rhythm disruption in neuropsychiatric illness. Curr Opin Neurobiol 2013;23:888-894.

39. McClung CA. How might circadian rhythms control mood? Let me count the ways. Biol Psychiatry 2013;74:242-249.

40. DeWeerdt S. Mood disorders: the dark night. Nature 2013;497:S14-S15.

41. Nock MK, Hwang I, Sampson NA, Kessler RC. Mental disorders, comorbidity and suicidal behavior: results from the National Comorbidity Survey Replication. Mol Psychiatry 2010;15:868-876.

42. Breslau N, Peterson EL, Schultz LR, Chilcoat HD, Andreski P. Major depression and stages of smoking. A longitudinal investigation. Arch Gen Psychiatry 1998;55:161-166.

43. Simon GE, Hunkeler E, Fireman B, Lee JY, Savarino J. Risk of suicide attempt and suicide death in patients treated for bipolar disorder. Bipolar Disord 2007;9:526-530.

44. Oquendo MA, Galfalvy H, Russo S, Ellis SP, Grunebaum MF, Burke A, et al. Prospective study of clinical predictors of suicidal acts after a major depressive episode in patients with major depressive disorder or bipolar disorder. Am J Psychiatry 2004:161:1433-1441.

45. Goldstein BI, Birmaher B, Axelson DA, Goldstein TR, Esposito-Smythers C, Strober MA, et al. Significance of cigarette smoking among youths with bipolar disorder. Am $J$ Addict 2008; 17:364-371.

46. Zarate CA Jr, Singh JB, Carlson PJ, Brutsche NE, Ameli $\mathrm{R}$, Luckenbaugh $\mathrm{DA}$, et al. A randomized trial of an $N$-methyl-D-aspartate antagonist in treatment-resistant major depression. Arch Gen Psychiatry 2006;63:856-864.

47. Phelps LE, Brutsche N, Moral JR, Luckenbaugh DA, Manji HK, Zarate CA Jr. Family history of alcohol dependence and initial antidepressant response to an N-methyl-Daspartate antagonist. Biol Psychiatry 2009;65:181-184.

48. aan het Rot M, Collins KA, Murrough JW, Perez AM, Reich DL, Charney DS, et al. Safety and efficacy of repeated-dose intravenous ketamine for treatment-resistant depression. Biol Psychiatry 2010;67:139-145.

49. Diazgranados N, Ibrahim L, Brutsche NE, Newberg A, Kronstein $\mathrm{P}$, Khalife $\mathrm{S}$, et al. A randomized add-on trial of an $\mathrm{N}$-methyl-D-aspartate antagonist in treatment-resistant bipolar depression. Arch Gen Psychiatry 2010;67:793-802.

50. Valtonen H, Suominen K, Mantere O, Leppämäki S, Arvilommi P, Isometsä ET. Suicidal ideation and attempts in bipolar I and II disorders. J Clin Psychiatry 2005;66: 1456-1462.

51. Galfalvy H, Oquendo MA, Carballo JJ, Sher L, Grunebaum MF, Burke A, et al. Clinical predictors of suicidal acts after major depression in bipolar disorder: a prospective study. Bipolar Disord 2006;8:586-595.

52. Marangell LB, Bauer MS, Dennehy EB, Wisniewski SR, Allen MH, Miklowitz DJ, et al. Prospective predictors of suicide and suicide attempts in 1,556 patients with bipolar disorders followed for up to 2 years. Bipolar Disord 2006; 8:566-575.

53. Malone KM, Waternaux C, Haas GL, Cooper TB, Li S, Mann JJ. Cigarette smoking, suicidal behavior, and serotonin function in major psychiatric disorders. Am J Psychiatry 2003; 160:773-779.

54. Dalley JW, Roiser JP. Dopamine, serotonin and impulsivity. Neuroscience 2012;215:42-58.
55. Brown RM, Kupchik YM, Kalivas PW. The story of glutamate in drug addiction and of $N$-acetylcysteine as a potential pharmacotherapy. JAMA Psychiatry 2013;70:895897.

56. Kalivas PW, Lalumiere RT, Knackstedt L, Shen H. Glutamate transmission in addiction. Neuropharmacology 2009; 56(Suppl 1):169-173.

57. Gipson CD, Reissner KJ, Kupchik YM, Smith AC, Stankeviciute N, Hensley-Simon ME, et al. Reinstatement of nicotine seeking is mediated by glutamatergic plasticity. Proc Natl Acad Sci U S A 2013;110:9124-9129.

58. Stockings E, Bowman J, McElwaine K, Baker A, Terry M, Clancy R, et al. Readiness to quit smoking and quit attempts among Australian mental health inpatients. Nicotine Tob Res 2013;15:942-949.

59. Geracioti TD Jr, West SA, Baker DG, Hill KK, Ekhator NN, Wortman MD, et al. Low CSF concentration of a dopamine metabolite in tobacco smokers. Am J Psychiatry 1999; 156:130-132.

60. Evins AE, Cather C, Pratt SA, Pachas GN, Hoeppner SS, Goff DC, et al. Maintenance treatment with varenicline for smoking cessation in patients with schizophrenia and bipolar disorder: a randomized clinical trial. JAMA 2014;311:145-154.

61. Berk M, Kapczinski F, Andreazza AC, Dean OM, Giorlando $\mathrm{F}$, Maes $\mathrm{M}$, et al. Pathways underlying neuroprogression in bipolar disorder: focus on inflammation, oxidative stress and neurotrophic factors. Neurosci Biobehav Rev 2011;35: 804-817.

62. Kalivas PW. The glutamate homeostasis hypothesis of addiction. Nat Rev Neurosci 2009;10:561-572.

63. Gipson CD, Kupchik YM, Kalivas PW. Rapid, transient synaptic plasticity in addiction. Neuropharmacology 2014; 76 Pt B:276-286.

64. Nunes SO, Vargas HO, Prado E, Barbosa DS, de Melo LP, Moylan S, et al. The shared role of oxidative stress and inflammation in major depressive disorder and nicotine dependence. Neurosci Biobehav Rev 2013;37:1336-1345.

65. Berk M, Williams LJ, Jacka FN, O'Neil A, Pasco JA, Moylan S, et al. So depression is an inflammatory disease, but where does the inflammation come from? BMC Med 2013;11:200-216.

66. Vargas HO, Nunes SO, de Castro MR, Vargas MM, Barbosa DS, Bortolasci CC, et al. Oxidative stress and inflammatory markers are associated with depression and nicotine dependence. Neurosci Lett 2013;544:136-140.

67. Haslemo T, Eikeseth PH, Tanum L, Molden E, Refsum H. The effect of variable cigarette consumption on the interaction with clozapine and olanzapine. Eur J Clin Pharmacol 2006;62:1049-1053.

68. Dani JA, De Biasi M. Cellular mechanisms of nicotine addiction. Pharmacol Biochem Behav 2001;70:439-446.

69. Laviolette SR, van der Kooy D. The neurobiology of nicotine addiction: bridging the gap from molecules to behaviour. Nat Rev Neurosci 2004;5:55-65.

70. Hutchison KE, Rutter MC, Niaura R, Swift RM, Pickworth WB, Sobik L. Olanzapine attenuates cue-elicited craving for tobacco. Psychopharmacology (Berl) 2004;175:407-413.

71. Caskey NH, Jarvik ME, Wirshing WC. The effects of dopaminergic D2 stimulation and blockade on smoking behavior. Exp Clin Psychopharmacol 1999;7:72-78.

72. Hagman BT, Delnevo CD, Hrywna M, Williams JM. Tobacco use among those with serious psychological distress: results from the national survey of drug use and health, 2002. Addict Behav 2008;33:582-592. 
73. Warner KE, Burns DM. Hardening and the hard-core smoker: concepts, evidence, and implications. Nicotine Tob Res 2003;5:37-48.

74. Pattanayak RD, Sagar R, Jain R. Perceived health risks, attitude and readiness to quit tobacco among euthymic bipolar disorder patients in regular contact with mental health services: an exploratory study from India. J Ment Health 2012;21:83-90.

75. Weinberger AH, Vessicchio JC, Sacco KA, Creeden CL, Chengappa KN, George TP. A preliminary study of sustained-release bupropion for smoking cessation in bipolar disorder. J Clin Psychopharmacol 2008;28:584-587.

76. Wu BS, Weinberger AH, Mancuso E, Wing VC, Haji-Khamneh B, Levinson AJ, et al. A preliminary feasibility study of varenicline for smoking cessation in bipolar disorder. J Dual Diagn 2012;8:131-132.

77. Taylor G, McNeill A, Girling A, Farley A, Lindson-Hawley $\mathrm{N}$, Aveyard P. Change in mental health after smoking cessation: systematic review and meta-analysis. BMJ 2014; 348:g1151.

78. Fiore M, Panel G. Treating tobacco use and dependence: 2008 update. Rockville, MD:Department of Health and Human Services;2008.

79. El-Guebaly N, Cathcart J, Currie S, Brown D, Gloster S. Smoking cessation approaches for persons with mental illness or addictive disorders. Psychiatr Serv 2002;53:1166-
1170.

80. Castro MR, Matsuo T, Nunes SO. Clinical characteristics and quality of life of smokers at a referral center for smoking cessation. J Bras Pneumol 2010;36:67-74.

81. Kerr S, Woods C, Knussen C, Watson H, Hunter R. Breaking the habit: a qualitative exploration of barriers and facilitators to smoking cessation in people with enduring mental health problems. BMC Public Health 2013;13:221.

82. Cook BL, Wayne GF, Kafali EN, Liu Z, Shu C, Flores M. Trends in smoking among adults with mental illness and association between mental health treatment and smoking cessation. JAMA 2014;311:172-182.

83. Pasco JA, Williams LJ, Jacka FN, Ng F, Henry MJ, Nicholson GC, et al. Tobacco smoking as a risk factor for major depressive disorder: population-based study. $\mathrm{Br} J$ Psychiatry 2008;193:322-326.

84. Lawrence D, Hafekost J, Hull P, Mitrou F, Zubrick SR. Smoking, mental illness and socioeconomic disadvantage: analysis of the Australian National Survey of Mental Health and Wellbeing. BMC Public Health 2013;13:462.

85. Caggiula AR, Donny EC, White AR, Chaudhri N, Booth S, Gharib MA, et al. Cue dependency of nicotine self-administration and smoking. Pharmacol Biochem Behav 2001; 70:515-530.

86. Berk M. Should we be targeting smoking as a routine intervention? Acta Neuropsychiatr 2007;19:131-132. 